\title{
FIB milling of polymer ceramic nanocomposites: far-reaching thermal artefacts and application to analysis of corrosion barrier coatings
}

Konrad Rykaczewski ${ }^{1}$, Daniel G. Mieritz ${ }^{2}$, Minglu Liu ${ }^{1, b)}$, Yuanyu Ma ${ }^{1}$, Erick B. Iezzi ${ }^{3}$, Xiaoda Sun ${ }^{1}$, Liping P. Wang ${ }^{1}$, Kiran N. Solanki ${ }^{1}$, Don K. Seo ${ }^{2}$, and Robert Y. Wang ${ }^{1}$

1. School for Engineering of Transport, Matter and Energy, Arizona State University, Tempe, AZ, US

2. Department of Chemistry and Biochemistry, Arizona State University, Tempe, AZ, US

3. Naval Research Laboratory, Chemistry Division, Washington, DC, US

FIB-SEMs are commonly used to characterize composite materials [1], however, their application to analysis of polymeric corrosion barrier coatings has been limited. This technique can be used to quantify 3D distributions of pigment particles and pores in pristine and corroded samples. Resistance of the coatings to penetration by water and dissolved ions is strongly affected by both of these geometrical features but their influence is typically quantified in effective terms using macroscopic measurements [2-3]. Chen et al.[2] recently quantified the 3D distribution of aluminum flakes dispersed in epoxy using serial block-face SEM imaging and x-ray tomography and used this information to theoretically predict barrier properties of the coating. As oppose to slicing using an ultramicrotome, FIB can be used to mill a smooth cross section of a pigmented paint without risk of artefacts arising from heterogeneous properties of the sample such as dislodging of particles by the microtome blade.

One of the main concerns that arise when using a FIB-SEM to characterize polymeric materials is the possibility of ion beam heating induced damage [4-5]. Previously thermal damage stemming from a significant temperature rise at the point of ion beam impact has been described. This temperature rise is proportional to ratio of the beam power and thermal conductivity of the material and for polymers characterized by low thermal conductivity could reach over thousand of degrees Celsius [5]. The degree of sample heating can be decreased using cryogenic cooling of the sample, low ion beam current during milling, and intelligent navigation of the ion beam [4-7].

In this work FIB-SEM is used to study surface and internal morphology of polysiloxane corrosion barrier coatings with various concentrations of pigment nanoparticles prior to and at different stages of accelerated corrosion tests. These novel coatings are potential candidates for replacing silicone alkyds, which are currently used to paint the topside of naval surface vessels. The single and two component polysiloxane coatings are hydrophobic and have enhanced cleanability, gloss retention, hardness, and color-stability as compared to the silicone alkyds [8-9]. It is shown that room temperature FIB cross sectioning of pure polysiloxane can be done with a wide range of ion beam currents ( 1 to $20 \mathrm{nA}$ ) without causing any significant damage the exposed section (see Figure 1a and 1b). In contrast, FIB milling of the pigmented polysiloxane coating with ion beam current higher than $1 \mathrm{nA}$ leads to an unexpected mode of severe polymer damage. Specifically, it is shown that FIB milling of such polymer-ceramic nanoparticle composites can lead to formation of multiple voids within the substrate as far as $5 \mu \mathrm{m}$ away from the ion beam impact (see Figure 1c and 1d). Using systematic experimentation coupled with heat transfer modeling, it is shown that the primary void formation occurs due to decomposition and vaporization of the polymer around ion beam heated pigment nanoparticles (see schematic in Figure 1e). The primary void enlargement and formation of secondary voids likely occurs due to mechanical damage of the polymer induced by the entrapped vapors that could be pressurized up to 100 MPa [10]. Similarly, secondary voids could form via cracking and seepage of the high-pressure vapors along weaker parts of the composite such as neighboring particle-polymer interfaces. This work demonstrates that processes occurring during FIB-milling of pure polymers and their composites with strongly 
heterogeneous thermal and mechanical properties can differ dramatically and care must be taken to avoid confusion of processing induced artefacts and genuine sample features.

\section{References:}

[1] F. Stevie, L. Giannuzzi, and B. Prenitzer Introduction to focused ion beams: instrumentation, theory, techniques and practice. (2005) New York: Springer.

[2] B., Chen, et al. Scientific Reports, 3, (2013), 1177.

[3] T., Nguyen, et al. Journal of Coatings Technology, 68, (1996) 45-56.

[4] C. A. Volkert and A. M. Minor MRS Bulletin, 32, (2007) 389-399.

[5] N. D., Bassim, et al Journal of Microscooy, 245, (2012) 288-301.

[6] R. Schmied et al. Physical Chemistry Chemical Physics, 16, (2014) 6153-6158.

[7] A. Orthacker et al. Physical Chemistry Chemical Physics, 16, (2014) 1658-1666.

[8] E. B. Iezzi, et al. Naval Research Laboratory Review (2013).

[9] E. B. Iezzi, E. B. US Patent US 8133964 B2 (2012).

[10] K. Rykaczewski et al. Journal of Microscopy (2015), DOI: 10.1111/jmi.12367

[11] KR, KNS, and LPW acknowledge funding from US Department of Defense under Technical Corrosion Collaboration Program administered by the US Air Force Academy through grant no.

FA7000-14-2-0015. Authors gratefully acknowledge the use of facilities with the LeRoy Eyring Center for Solid State Science at Arizona State University.
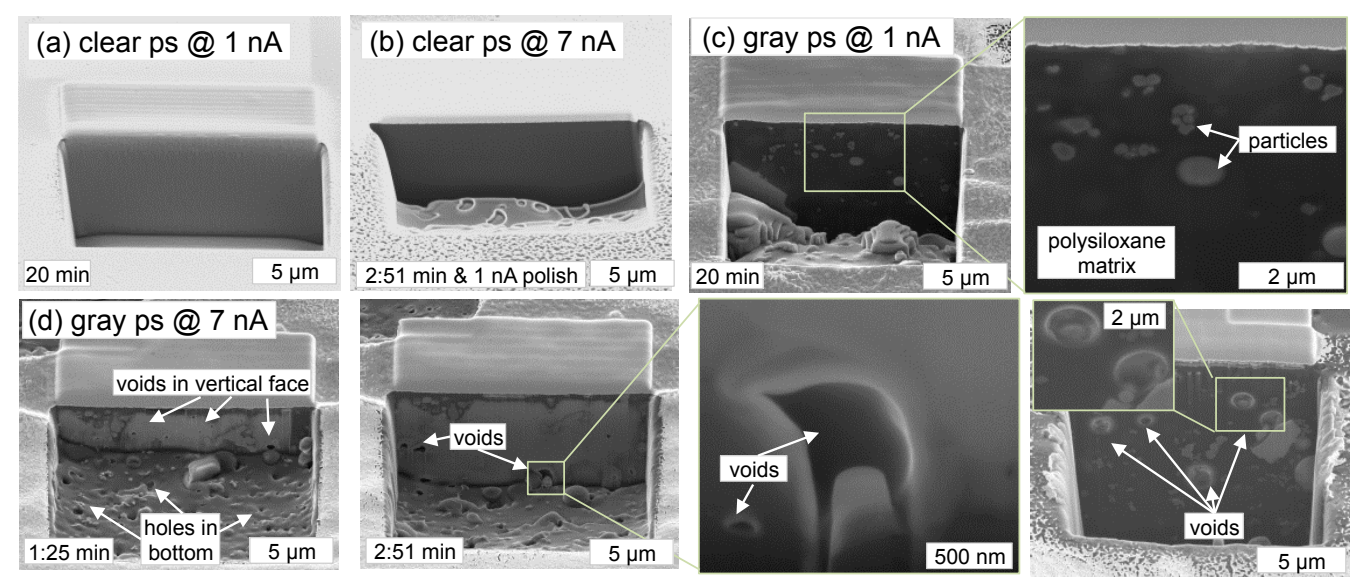

(e)

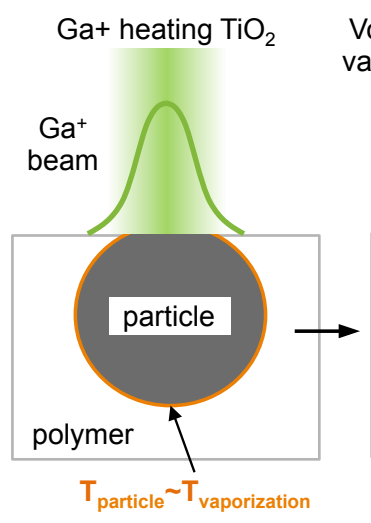

Void formation via polymer Void growth and spreading via vaporization at $\mathrm{TiO}_{2}$ surface vaporization of polymer by hot gas and/or polymer yielding to the
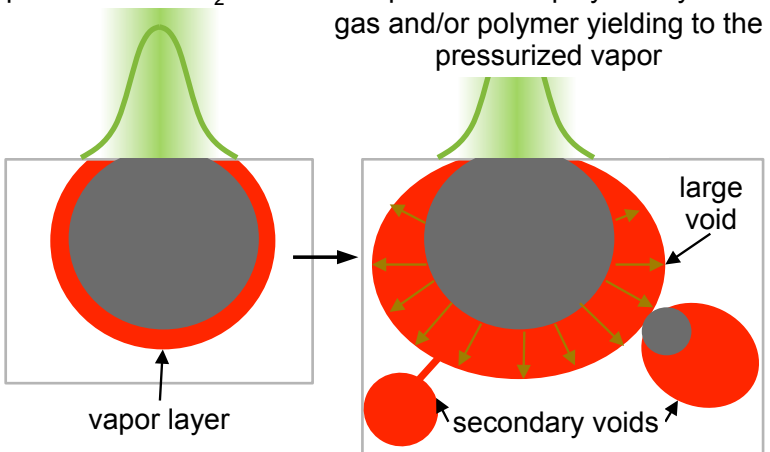

Figure 1. $52^{\circ}$ tilt SEM images of clear polysiloxane FIB milled at (a) $1 \mathrm{nA}$ and (b) $7 \mathrm{nA}$ and of the haze gray polysiloxane FIB milled at (c) $1 \mathrm{nA}$ and (d) $7 \mathrm{nA}$; and (e) Schematic of the proposed thermal route of void formation. 